\title{
Nanoemulsion as a topical delivery system of antipsoriatic drugs
}

\begin{abstract}
Psoriasis is one of the most common skin diseases, affecting $2-5 \%$ of the world's population. It is a skin autoimmune disorder, resulting in an excessive growth and aberrant differentiation of keratinocytes. Psoriasis is an incurable lifetime disease which can only be controlled and relieved through medication. Various approaches have been explored to treat the disease. Treatment of psoriasis includes topical therapy, systemic therapy and phototherapy. Topical therapy is the first line treatment and it is the most practical medication method for psoriasis patients. However, the conventional topical treatments such as gel and cream have low efficiency, poor cosmetic and aesthetic appeal, leading to poor patient compliance or adherence, while systemic and photo therapy produce significant adverse side effects. Nanoemulsion is defined as an emulsion system consisting of oil, surfactant, and water with an isotropic, transparent (or translucent) appearance. The emulsion droplet size is defined to be less than $200 \mathrm{~nm}$. Nonetheless, if the emulsion has low surfactant content and is kinetically stable, a size of less than $500 \mathrm{~nm}$ can be accepted as nanoemulsion. A small droplet size would enhance the delivery and penetration of a drug through the psoriasis skin layer. There has been a growing interest in using nanoemulsions in topical applications, due to their high stability and their optical transparency or translucency, which make them good and very dermatologically attractive. A good selection of oils and surfactants would enhance the transdermal treatment efficacy. This review highlights the potential of drug-loaded nanoemulsions for the treatment of psoriasis towards achieving better efficacy and eliminating side effects.
\end{abstract}

Keyword: Nanoemulsion; Topical delivery system; Antipsoriatic drugs 